\title{
A New Hope for Cancer Therapy with Liquid Knife \& Immuno Therapy: UMIPIC
}

\author{
Baofa Yu* \\ Beijing Baofa Cancer Hospital, China
}

*Corresponding author: Baofa Yu, Beijing Baofa Cancer Hospital, Beijing, China.

Received Date: March 15, 2020

Published Date: March 20, 2020

\section{Short Communication}

Recent years cancer immunological therapy is getting very popular and many new drug have been approved by FDA like PD1 and PDI-1, however, in clinical practice of cancer treatment, it looks very limited efficacy for advanced cancer, so that physician started to use comprehensive plan by combination chemotherapy with PD1 as a novel strategy with a better clinical benefit. Since chemotherapy always produce the side effect like loss hair, vomit and neutropenia. Because of the extremely toxic side effects, many cancer patients cannot successfully complete a complete course of chemotherapy, and some even die from the side effects of chemotherapy due to a patient's poor tolerance. The extreme side effects of anti-cancer drugs are often caused by the poor target specificity of such drugs regarding the tumor in the patient's body. The drugs circulate through most normal organs of patients as well as the intended target tumors (less than $5 \%$ of the drug reach the tumor), while over $95 \%$ of the drug circulates through the whole body of the patient.

The more and more cancer patients do not like to choice to chemotherapy, it is pushing the oncologists to be in the embarrassed situation to suggest the therapy for the patient who lost surgery opportunity, in the fact for most late stages of cancer, how many choice our oncologists can give? a few choice, today I do like to introduce one treatment which is suitable for the most of latte stages of solid tumor with benefit, UMIPIC, ultra-minimum incision personalized intratumorally chemoimmunotherapy.

UMIPIC is a unique eclectic approach for cancer treatment utilizing the intratumorally injection of a combination of chemotherapy drugs (including Dox and Ara-C), coagulant agent oxidant to maintain the chemo agents at the injection site for a longer period of time, and a hapten for hapenation with intracellular tumor-associated antigens to stimulate the patient's immune response while the tumor is been de-bulking like liquid surgery knife. Safer and more aggressive (higher dose) administration of toxic chemotherapy drugs that go directly into a tumor site with slow release, is an obvious and beneficial alternative to systemic treatment. The local retention afforded by intratumoral administration results in slowed and/or reduced entry of drug into the systemic circulation, minimizing exposure of distant tissues to the drug, and thus, resulting in a lower incidence of systemic side effects a more tumor killed. So far, there are 56 clinical trials found for intratumoral chemotherapy (ITCT) by visiting the website at the following: https://clinicaltrials.gov. But there is not one integrated with immune therapy and surgery knife to action like de-bulk.

In 1994, Dr. Yu developed the new concept of using the tumor itself as a drug carrier. Injection of anti-cancer drug ethanol saturated liquid into the tumor can generate a kind of intratumoral autologous therapeutic coagulum which can function as an antitumor drug depot. This autologous therapeutic coagulum can sustain or store anticancer drug in tumor and the surrounding tumor tissues to kill the tumor cells that have not been killed through ethanol coagulation treatment. It makes a fitting complement for the inadequacy of pure ethanol tumor treatment. A study of retaining drug in injected tumor demonstrated that the retention half-life of anticancer drug Ara-C in the tumor was 160 minutes following depot injection compared to only 6 minutes following 
intratumoral injection of Ara-C aqueous solution, thus increasing the drug retention by approximately 27 times [1]. The problem of using ethanol is that is is limited by the dosage for the patient and is also limited by tumor size (when the volume of tumor is large, it can dilute ethanol making it inefficient). Now Dr. Yu found oxidant is good role to replace the ethanol to coagulation of tumor as a drug carrier for slow releasing [2].

From 2003 to 2006, Dr. Yu also published many papers showing that the combination of intratumoral drug with hapten modification improves the immunogenicity of tumor cells, effectively inducing or activating body's antitumor immune response and had 276 patients with cancer reported [3-5]. It indicates that when hapten is added to the UMIPIC, it plays an important role in stimulating immune response.

The UMIPIC is comprised of commercially available drugs for intratumoral injection which includes oxidant as coagulant, chemotherapy drugs and hapten. Intratumoral injection with UMIPIC, it produces its antitumor role in the following aspects:

The first aspect is coagulation by the oxidant. "Tumor coagulation" refers the process by which the blood clots to form solid masses, or clots, and their components and extracellular matrix are transformed into a kind of soft, semi-solid, or solid block. This transformation is induced by oxidation, which makes openings in the membrane of tumor cells. This creates higher permeability of membrane that allows the drugs to penetrate into tumor cells and eventually leads to the death of coagulated tumor cells and enhancement of the cancer drug entering agglomerated tumor cells.

The second aspect is the concentration and sustainability of the drugs, the two key elements: Drug dosage and amount of time for destruction of cancer cells. Tumor coagulation also creates a "drug depot" which not only increases local drug action concentration (dozens or even hundreds of times more than the normal concentration by intravenous chemotherapy) to kill the tumor, but also retains the drugs within the tumor and gradually releases them from inside to the outside to kill residual tumor cells around tumor tissue. This "drug depot" not only extends drug action time in the tumor, but also prevents the leakage of anti-cancer drugs from the tumor, and lowers systemic drug concentration, toxicity and side effects.

The third aspect is stimulation of immune response. Tumor cells killed by the tumor coagulation effect and the chemotherapeutic agents could release intracellular proteins including tumorassociated antigens, which may already interact with hapten in that active reaction with tumor oxidation. The tumor antigens induce a personalized systemic immune response and the haptenation of tumor antigens could further stimulate immune response, thereby eliminating recurring or metastatic tumor cells.
The schematic diagram is shown with the function of components in UMIPIC and the Procedure of UMIPIC: Guided by $\mathrm{CT}$, find the optimal route and angle for introducing the needle intratumoral, the needle is inserted into the tumor, connected to the inflator, the regimens of UMIPIC were slowly delivered into the tumor; a high pressure supplied by the inflator; the solution (UMIPIC) can penetrate into the extracellular matrix of tumor and facilitate forced diffusion. Same injection could be repeated to same tumor or other tumor several times according to evaluating by CT and physician or investigator brochures [6-9].

In the past years, UMIPIC treated lung cancer, median overall survival was 11.23 months in the UMIPIC (test) group and 5.62 months in the ITCT (control) group $(\mathrm{P}<0.01)$. The 6-month and 1-year survival rates of the UMIPIC and ITCT groups were $76.36 \%$ versus $45.23 \%(\mathrm{P}<0.01)$ and $45.45 \%$ versus $23.81 \%(\mathrm{P}<0.05)$, respectively. Two cycles of UMIPIC treatment $(\mathrm{N}=19)$ conferred a significant survival benefit compared with two cycles of ITCT $(\mathrm{N}=29)$; significant benefits in survival time were also found with UMIPIC $(\mathrm{N}=20)$ compared with ITCT $(\mathrm{N}=13)$ when both were utilized without hapten treatment.

Also UMIPIC for liver cancer with good result: the benefit rates (complete response + partial response + stable disease) were $78.68 \%$ and $81.52 \%$ in the UMIPIC and ITCT groups, respectively, with no statistically significant difference; however, the median overall survival was 7 months for UMIPIC (test) and 4 months for ITCT (control), respectively $(\mathrm{P}<0.01)$. The 6-month and 1-year survival rates for UMIPIC and ITCT were $58.88 \%$ vs $32.3 \%$ and $30.37 \%$ vs $13.6 \%$, respectively $(\mathrm{P}<0.01)$. Single and multiple UMIPIC revealed significant improvement in overall survival compared to that of ITCT.

In the past years, UMIPIC treated pancreatic cancer, for single drug, median survival was 6.45 months for UMIPIC-S vs 4.98 months for ITCT-S, $(\mathrm{P}<0.05)$, one-year survival rate was $28 \%$ for UMIPIC-S vs $5 \%$ for ITCT-S $(\mathrm{P}<0.05)$. For double drugs, median survival was 15.5 months for UMIPIC-D vs 3 months in ITCT-D $(\mathrm{P}<0.01)$. The 6-month survival rate was $76.67 \%$ for UMIPIC-D vs $18.18 \%$ for ITCT-D $(\mathrm{P}<0.01)$ and 1-year survival rate for $56.67 \%$ UMIPIC-D vs $9.09 \%$ ITCT $(\mathrm{P}<0.01)$.

UMIPIC is a simple, clinically effective drug for a broad spectrum of tumors with minimal side effects through ultra-minimal invasive surgery under the guide of CT or ultrasound. In conclusion, UMIPIC provides a new method of decreasing tumor mass while boost the patient's own immunological power to fight against micro tumor cells in a specific and innovative manner, which is one of its advantages over any treatment. Another advantage is that it is not limited in terms of tumor size, number, or location in the lung, liver, pancreatic or any location of tumor. In future, it is possible that UMIPIC may overtake in the treatment of all stages of tumor in the 
lung, liver, pancreatic or any location of tumor. UMIPIC can take the place of surgery and chemotherapy or radiation therapy in patients who are not suited for surgery or chemotherapy. We hope to continue to investigate UMIPIC therapy with double cytotoxic drugs with double hapten under clinical study to improve effectiveness.

Today, it is time to think how to replace the surgery, chemotherapy and radiation therapy which produce a damage of patient with cancer, we believe UMIPIC has provided a new eclectic approach for the treatment of primary all solid tumor at anywhere of body, even pre surgery and during of operation. UMIPIC is safe, easy to operate, and reproducible with good benefit for all solid tumor.

\section{Acknowledgement}

None.

\section{Conflict of Interest}

No conflict of interest.

\section{References}

1. Yu B, Kim S (1994) Alcohol with Intratumoral Durg Injection and Pharmacokinetics of Drug After Intratumoral Injection: A New Concept of Intraturmoal Autologous Therapeutic Coagulum with Drug Depots. Journal of Current Oncology 1(2): 97-100.
2. Jieqiong B, Yu B (20074) Slow intra-tumor release of drugs on B16 melanoma in Mice. Journal of Shandong University (Health Sciences), 45(10): 988-991.

3. Yu B, Ma Z, Guan C (2003) Observation of 751 cases of malignant tumors treated with drug depot therapy. Journal of Shandong University (Health Sciences) 41: 14-18.

4. Baofa Yu, Han Y, Liu G (2003) Animal Experiment of Intratumoral Drug (ARA-C) Slow Release Depot Therapy. Journal of Shandong University (Health Science) 41(Suppl): 1-3.

5. Guan C, Yang G, Zhang J (2006) Observation of 276 cases of advanced liver tumors treated with drug depot therapy. Chinese Journal of Celiopathy 6(3): 160-162.

6. Yu B (2014) Interventional Oncology \& Chemoimmunotherapy: 1-9.

7. Yu B, Lu Y, Gao F, Jing P, Wei H, et al. (2015) Hapten-enhanced therapeutic effect in advanced stages of lung cancer by ultra-minimum incision personalized intratumoral chemoimmunotherapy. Lung Cancer: Target and Therapy 6: 1-11.

8. Gao F, Jing P, Liu J, Lu Y, Zhang P, et al. (2015) Hapten-enhanced overall survival time in advanced hepatocellular carcinoma by ultra-minimum incision personalized intratumoral chemoimmunotherapy. J Hepatocell Carcinoma 2: 1-12.

9. Jing P, Li J, Gao F, Lu Y, liu J, et al. (2015) Use of hapten combinded Catatonic drugs for Enhancing Therapeutic Effect in Advanced Stages of Pancreatic Cancer. Journal of Liver Research, Disorders \& Therapy 1(3): 13-23. 